\title{
METABELIAN LIE POWERS OF GROUP REPRESENTATIONS
}

\author{
R. M. BRYANT, R. STÖHR and R. ZERCK
}

(Received 14 November 1990)

Communicated by H. Lausch

\begin{abstract}
Any representation of a group $G$ on a vector space $V$ extends uniquely to a representation of $G$ on the free metabelian Lie algebra on $V$. In this paper we study such representations and make some group-theoretic applications.
\end{abstract}

1991 Mathematics subject classification (Amer. Math. Soc.): 20 C 15, 20 F 40.

\section{Introduction}

Let $K$ be a commutative ring with identity and let $X$ be a set. We write $S^{K}(X)$ for the free commutative associative $K$-algebra with identity on $X$ (equal to the polynomial ring $K[X]), L^{K}(X)$ for the free Lie algebra over $K$ on $X$, and $M^{K}(X)$ for the free metabelian Lie algebra over $K$ on $X$. (Thus $M^{K}(X)$ is isomorphic to $L^{K}(X)$ factored out by its second derived algebra. See $[2,4,5,15]$ for basic material concerning Lie algebras.) Let $V$ be a free $K$-module such that $X$ is a basis of $V$. (All our modules will be left unital modules.) Then $V$ can be identified with the $K$-submodule of $S^{K}(X)$ spanned by $X$; and the same can be done for $L^{K}(X)$ and $M^{K}(X)$. We define $\mathbf{S} V=S^{K}(X), \mathbf{L} V=$ $L^{K}(X)$ and $\mathbf{M} V=M^{K}(X)$. (With $V$ identified in the way described, these algebras are independent of the choice of basis of $V$.) $\mathbf{S} V$ has a $K$-module decomposition $\mathbf{S} V=\bigoplus_{n \geq 0} \mathbf{S}_{n} V$ where $\mathbf{S}_{n} V$ is the submodule spanned by all

(C) 1994 Australian Mathematical Society 0263-6115/94 \$A2.00+0.00 
monomials $x_{i_{1}} \ldots x_{i_{n}}$ with $x_{i_{1}}, \ldots, x_{i_{n}} \in X$. Similarly $\mathbf{L} V=\bigoplus_{n \geq 1} \mathbf{L}_{n} V$ and $\mathbf{M} V=\bigoplus_{n \geq 1} \mathbf{M}_{n} V$ where $\mathbf{L}_{n} V$ and $\mathbf{M}_{n} V$ are spanned by the left-normed Lie monomials $\left[x_{i_{1}}, \ldots, x_{i_{n}}\right]$ with $x_{i_{1}}, \ldots, x_{i_{n}} \in X$. (We use square-bracket notation for Lie products.)

For any $K$-module $V$ (not necessarily free) we write $\mathrm{GL}_{K}(V)$ or $\mathrm{GL}(V)$ for the group of all $K$-module automorphisms of $V$. If $V$ is free then for each $g \in \mathrm{GL}(V)$ the action of $g$ on $V$ extends (uniquely) to give algebra automorphisms of $\mathbf{S} V$, $\mathbf{L} V$ and $\mathbf{M} V$ : for example, $g\left[x_{i_{1}}, \ldots, x_{i_{n}}\right]=\left[g x_{i_{1}}, \ldots, g x_{i_{n}}\right]$. Thus $\mathbf{S} V, \mathbf{L} V$ and $\mathbf{M} V$ become modules for the group algebra $K \mathrm{GL}(V)$ in which each element of GL( $V)$ acts as an algebra automorphism. Clearly each $\mathbf{S}_{n} V, \mathbf{L}_{n} V$ and $\mathbf{M}_{n} V$ is a $K \mathrm{GL}(V)$-submodule. More generally, if $H$ is any group and $V$ is a $K H$ module which is free as a $K$-module then the representation $H \rightarrow \mathrm{GL}(V)$ gives a $K H$-module structure to $\mathbf{S} V, \mathbf{L} V$ and $\mathbf{M} V$.

THEOREM A. Let $V$ be a finite dimensional vector space of dimension at least 2 over a field $K$ and let $G$ be a finite subgroup of $\mathrm{GL}(V)$. Then, for all $n \geq 1$, there exists $t \geq n$ such that $\mathbf{M}_{n} V \oplus \cdots \oplus \mathbf{M}_{t} V$ has a regular $K G$-submodule.

A similar result for $\mathbf{S} V$ is well known. (We shall give a simple proof in Section 3.) An analogue of Theorem A for $\mathbf{L} V$ was proved by Bryant and Kovács [8] where the result was applied to the study of automorphism groups of finite $p$-groups ( $p$ a prime). If $P$ is a finite $p$-group and $\Phi(P)$ denotes the Frattini subgroup of $P$ then every automorphism of $P$ induces an automorphism of $P / \Phi(P)$, giving a homomorphism $\pi: \operatorname{Aut}(P) \rightarrow \operatorname{Aut}(P / \Phi(P))$ from the automorphism group of $P$ to that of $P / \Phi(P)$. We may regard $P / \Phi(P)$ as a vector space over $\mathbb{F}_{p}$, the field of $p$ elements: thus $\pi(\operatorname{Aut}(P))$ is a linear group over $\mathbb{F}_{p}$. In [8] it was shown that every linear group of finite dimension at least 2 over $\mathbb{F}_{p}$ arises from some $P$ in this way. Here we use Theorem A to show that $P$ may be taken to be metabelian.

THEOREM B. Let $p$ be a prime number. For every linear group $G$ of finite dimension at least 2 over $\mathbb{F}_{p}$ there exists a finite metabelian p-group $P$ such that $G$ is isomorphic as linear group to the image of $\pi: \operatorname{Aut}(P) \rightarrow \operatorname{Aut}(P / \Phi(P))$.

Our other results are concerned with the case where $K$ is a field of characteristic 0 and $V$ is a finite dimensional vector space over $K$. Thus the $K \mathrm{GL}(V)$-modules $\mathbf{S}_{n} V, \mathbf{L}_{n} V$ and $\mathbf{M}_{n} V$ are all finite dimensional. For any finite dimensional $K \mathrm{GL}(V)$-module $W$ we write $\chi_{W}$ for the character of $\mathrm{GL}(V)$ 
on $W$. A formula for $\chi_{\mathbf{L}_{n} V}$ was given by Brandt [6]. Here we shall give formulae for the 'generating functions' of $\chi_{\mathbf{S}_{n} V}$ and $\chi_{\mathbf{M}_{n} V}$ (see Proposition 4.1).

We shall apply these formulae to the 'relation module' of a finite group. Let $F$ be a free group of finite rank and let $R$ be a normal subgroup of $F$ such that $G=F / R$ is finite. The relation module for $G$ is the derived factor group $R / R^{\prime}$ regarded as a $\mathbb{Z} G$-module ( $\mathbb{Z}$ the ring of integers) by means of conjugation: $(f R)\left(u R^{\prime}\right)=\left(f u f^{-1}\right) R^{\prime}$ for all $f \in F, u \in R$. This module is faithful for $G$ when $F$ is non-cyclic (see [3]). So, with $G$ regarded as a subgroup of $\mathrm{GL}_{\mathbb{Z}}\left(R / R^{\prime}\right)$, the algebras $\mathrm{S}\left(R / R^{\prime}\right), \mathbf{L}\left(R / R^{\prime}\right)$ and $\mathbf{M}\left(R / R^{\prime}\right)$ have the structure of $\mathbb{Z} G$-modules. By tensoring with $\mathbb{Q}$ (the field of rational numbers) we obtain a finite dimensional $\mathbb{Q} G$-module $V=\mathbb{Q} \otimes_{\mathbb{Z}}\left(R / R^{\prime}\right)$ and $\mathbb{Q} G$-modules $\mathbf{S} V$, $\mathbf{L} V$ and $\mathbf{M} V$. Several results about $\mathbf{L} V$ as $\mathbb{Q} G$-module, derived from Brandt's character formula, were obtained by Gupta, Laffey and Thomson [13]. Here we obtain some analogous results concerning $\mathbf{S} V$ and $\mathbf{M} V$.

In the statement of the following theorem, $|G|$ denotes the order of the finite group $G$ and $|g|$ denotes the order of the element $g$ of $G$. For non-negative integers $a$ and $b,(a, b)$ denotes the greatest common divisor of $a$ and $b$ and, when $a \geq b,\left(\begin{array}{l}a \\ b\end{array}\right)$ denotes the binomial coefficient $a ! /(b !(a-b) !)$.

THEOREM C. Let $F$ be a free group of finite rank $e \geq 2$ and let $R$ be a normal subgroup of $F$ such that $G=F / R$ is finite and $|G| \neq 1$. Let $V=\mathbb{Q} \otimes_{\mathbb{Z}}\left(R / R^{\prime}\right)$, and regard $V$ as $a \mathbb{Q} G$-module by means of conjugation. Let $m=1+(e-1)|G|$.

(i) Let $g \in G$ and write $q=|g|$. For all $n \geq 0$,

$$
\chi_{\mathbf{S}_{n} v}(g)=\left(\begin{array}{c}
((m-1) / q)+[n / q] \\
{[n / q]}
\end{array}\right)
$$

where $[n / q]$ is the greatest integer not exceeding $n / q$.

(ii) Let $g \in G$ and write $q=|g|$. For all $n \geq 2$,

$$
\chi_{\mathbf{M}_{n} v}(g)=\left\{\begin{array}{cl}
0 & \text { if } q \nmid n, \\
-\left(\begin{array}{c}
(m+n-q-1) / q \\
n / q
\end{array}\right) & \text { if } q \mid n \text { and } q \neq 1, \\
(n-1)\left(\begin{array}{c}
m+n-2 \\
n
\end{array}\right) & \text { if } q=1 .
\end{array}\right.
$$

(iii) For $n \geq 2$, the multiplicity of the one-dimensional trivial $\mathbb{Q} G$-module 
in $\mathbf{M}_{n} V$ is

$$
d_{n}=\frac{1}{|G|}\left\{(n-1)\left(\begin{array}{c}
m+n-2 \\
n
\end{array}\right)-\sum_{\substack{q \mid(n,|G|) \\
q \neq 1}} v(q)\left(\begin{array}{c}
(m+n-q-1) / q \\
n / q
\end{array}\right)\right\},
$$

where $\nu(q)$ denotes the number of elements of $G$ with order $q$. Furthermore $d_{n}$ is the multiplicity in $\mathbf{M}_{n} V$ of the regular $\mathbb{Q} G$-module (that is, the greatest rank of a free $\mathbb{Q} G$-submodule of $\mathbf{M}_{n} V$ ).

(iv) If $n \geq 2$ and $(n,|G|)=1$ then $\mathbf{M}_{n} V$ is a free $\mathbb{Q} G$-module of rank

$$
\frac{1}{|G|}(n-1)\left(\begin{array}{c}
m+n-2 \\
n
\end{array}\right) \text {. }
$$

Theorem C has some group-theoretic consequences. Most of our notation for groups is standard but, to avoid confusion with Lie multiplication, we use round brackets for group commutators: $(g, h)=g^{-1} h^{-1} g h$. For $n \geq 1, \gamma_{n} H$ denotes the $n$th term of the lower central series of the group $H: \gamma_{1} H=H$ and $\gamma_{n+1} H=\left(\gamma_{n} H, H\right)$. Also, for $n \geq 1$, we write $\mu_{n} H=\left(\gamma_{n} H\right) H^{\prime \prime}$ (where $H^{\prime \prime}$ is the second derived group of $H$ ). Thus $H / \mu_{n} H$ is the largest factor group of $H$ which is both nilpotent of class $n-1$ and metabelian.

Let $F, R$ and $G=F / R$ be as before. The factor groups $\gamma_{n} R / \gamma_{n+1} R$ and $\mu_{n} R / \mu_{n+1} R$ may be regarded as $\mathbb{Z} G$-modules by means of conjugation. Thus $(f R)\left(u \gamma_{n+1} R\right)=\left(f u f^{-1}\right) \gamma_{n+1} R$ for all $f \in F, u \in \gamma_{n} R$, and similarly for $\mu_{n} R / \mu_{n+1} R$. The modules $\gamma_{n} R / \gamma_{n+1} R$ are the 'higher relation modules'. There are $\mathbb{Z} G$-module isomorphisms $\gamma_{n} R / \gamma_{n+1} R \cong \mathbf{L}_{n}\left(R / R^{\prime}\right)$ and $\mu_{n} R / \mu_{n+1} R \cong$ $\mathbf{M}_{n}\left(R / R^{\prime}\right)$ (see Section 4). In [13] results about $\mathbf{L}_{n}\left(\mathbb{Q} \otimes\left(R / R^{\prime}\right)\right)$ were used to obtain information about $\gamma_{n} R / \gamma_{n+1} R$. Here we use Theorem $\mathrm{C}$ to obtain information about $\mu_{n} R / \mu_{n+1} R$. For any group $H$ we write $Z(H)$ for the centre of $H$ and, for any $H$-module $W$, we write $W^{H}=\{w \in W: h w=w$ for all $h \in H\}$.

THEOREM D. Let $F, R$ and $G$ be as in Theorem C. Let $d_{1}=e$ and, for $n \geq 2$, let $d_{n}$ be defined as in Theorem $\mathrm{C}$ (iii).

(i) For $n \geq 1, Z\left(F / \mu_{n+1} R\right)=\left(\mu_{n} R / \mu_{n+1} R\right)^{G}$ and this is a free abelian group of rank $d_{n}$.

(ii) For $n \geq 1, \mu_{n} R /\left(\mu_{n} R, F\right) \mu_{n+1} R$ has torsion-free rank $d_{n}$.

In [14] Hannebauer and Stöhr studied the factors $\mu_{n} R /\left(\mu_{n} R, F\right) \mu_{n+1} R$ in the general case where $F / R$ is not necessarily finite. They showed in [14, 
Theorem 7.1] that, for $n \geq 2, \mu_{n} R /\left(\mu_{n} R, F\right) \mu_{n+1} R$ decomposes as the direct sum of a free abelian group $D_{n}$ and a torsion group $T_{n}$ which has exponent dividing $n$ if $n$ is odd and $2 n$ if $n$ is even. Theorem $\mathrm{D}$ (ii) above shows that, in the case where $F / R$ is finite, $D_{n}$ has rank $d_{n}$.

The organisation of the paper is as follows. Section 2 contains background information on Lie algebras and related topics. Theorems A and B will be proved in Section 3, and Theorems C and D in Section 4.

\section{Central series of subgroups and Lie algebras}

In this section we shall collect together information about the Lie algebras associated with certain descending series of subgroups of a group. Much of the material is well known, but we have re-cast it in a form suitable for our purposes. We have not attempted the difficult task of attributing individual results to their original sources. Suffice it to say that a major contribution was made by Lazard [16] and our account is greatly influenced by the account in Chapter VIII of Huppert and Blackburn's book [15].

Throughout this section $p$ will denote a fixed prime number. For any group $H$ and any positive integer $m, H^{m}$ denotes the subgroup of $H$ generated by all the $m$ th powers $h^{m}, h \in H$. For any Lie algebra $L, L^{\prime}$ denotes the derived algebra $[L, L]$ and $L^{\prime \prime}=\left[L^{\prime}, L^{\prime}\right]$.

Let $H$ be any group. We regard the lower central factors $\gamma_{n} H / \gamma_{n+1} H$ as $\mathbb{Z}$-modules and form the (restricted) direct sum

$$
\gamma H=\bigoplus_{n \geq 1} \gamma_{n} H / \gamma_{n+1} H
$$

As is well known, $\gamma H$ can be given the structure of a Lie ring (Lie algebra over $\mathbb{Z})$ by defining

$$
\left[u \gamma_{m+1} H, v \gamma_{n+1} H\right]=(u, v) \gamma_{m+n+1} H
$$

for all $u \in \gamma_{m} H, v \in \gamma_{n} H$ and all $m, n \geq 1$. (See, for example, [15, VIII.9.3].)

Similarly, the direct sum

$$
\tilde{\gamma} H=\bigoplus_{n \geq 1} \gamma_{n} H /\left(\gamma_{n} H\right)^{p} \gamma_{n+1} H
$$

can be given the structure of a Lie algebra over $\mathbb{F}_{p}$ by defining

$$
\left[u\left(\gamma_{m} H\right)^{p} \gamma_{m+1} H, v\left(\gamma_{n} H\right)^{p} \gamma_{n+1} H\right]=(u, v)\left(\gamma_{m+n} H\right)^{p} \gamma_{m+n+1} H
$$


for all $u \in \gamma_{m} H, v \in \gamma_{n} H$ and all $m, n \geq 1$. In fact $\tilde{\gamma} H \cong \mathbb{F}_{p} \otimes_{\mathbb{Z}} \gamma H$. Furthermore we write

$$
\tilde{\gamma}^{\prime} H=\bigoplus_{n \geq 2} \gamma_{n} H /\left(\gamma_{n} H\right)^{p} \gamma_{n+1} H
$$

Thus $\tilde{\gamma}^{\prime} H$ is a subalgebra of $\tilde{\gamma} H$.

For all $n \geq 1$ let

$$
\lambda_{n} H=\left(\gamma_{1} H\right)^{p^{n-1}}\left(\gamma_{2} H\right)^{p^{n-2}} \ldots\left(\gamma_{n} H\right) .
$$

Then (see [15, VIII.1.5]) $H=\lambda_{1} H \geq \lambda_{2} H \geq \ldots$, and, for all $m, n \geq 1$,

$$
\left(\lambda_{m} H, \lambda_{n} H\right) \leq \lambda_{m+n} H \quad \text { and } \quad \lambda_{n+1} H=\left(\lambda_{n} H\right)^{p}\left(\lambda_{n} H, H\right) .
$$

Thus $\lambda_{n} H / \lambda_{n+1} H$ is the largest factor group of $\lambda_{n} H$ which is an elementary abelian $p$-group centralized by $H$. The direct sum

$$
\lambda H=\bigoplus_{n \geq 1} \lambda_{n} H / \lambda_{n+1} H
$$

can be given the structure of a Lie algebra over $\mathbb{F}_{p}$ by defining

$$
\left[u \lambda_{m+1} H, v \lambda_{n+1} H\right]=(u, v) \lambda_{m+n+1} H
$$

for all $u \in \lambda_{m} H, v \in \lambda_{n} H$ and all $m, n \geq 1$. Furthermore we write

$$
\lambda^{\prime} H=\bigoplus_{n \geq 2}\left(H^{\prime} \cap \lambda_{n} H\right) \lambda_{n+1} H / \lambda_{n+1} H .
$$

Thus $\lambda^{\prime} H$ is a subalgebra of $\lambda H$.

Let $\mathbb{F}_{p}[\omega]$ be the ring of polynomials in an indeterminate $\omega$ over $\mathbb{F}_{p}$.

PROPOSITION 2.1. Let $H$ be any group.

(i) For $p \neq 2, \lambda H$ has the structure of an $\mathbb{F}_{p}[\omega]$-Lie algebra in which $\omega\left(u \lambda_{n+1} H\right)=u^{p} \lambda_{n+2} H$ for all $u \in \lambda_{n} H, n \geq 1$.

(ii) For all primes $p, \lambda^{\prime} H$ has the structure of an $\mathbb{F}_{p}[\omega]$-Lie algebra in which $\omega\left(u \lambda_{n+1} H\right)=u^{p} \lambda_{n+2} H$ for all $u \in H^{\prime} \cap \lambda_{n} H, n \geq 2$.

PROOF. It is easy to verify that, for all $n \geq 1$, there is a well defined map $\omega_{n}: \lambda_{n} H / \lambda_{n+1} H \rightarrow \lambda_{n+1} H / \lambda_{n+2} H$ given by $\omega_{n}\left(u \lambda_{n+1} H\right)=u^{p} \lambda_{n+2} H$ for all $u \in \lambda_{n} H$. If $p \neq 2$ or $n \neq 1$ then $(u v)^{p} \lambda_{n+2} H=u^{p} v^{p} \lambda_{n+2} H$ for all $u$, 
$v \in \lambda_{n} H$ : this follows from the congruence $(u v)^{p} \equiv u^{p} v^{p} \bmod \left(\gamma_{2} A\right)^{p}\left(\gamma_{p} A\right)$, where $A=\langle u, v\rangle$ (see [15, VIII.1.1a]). Thus, if $p \neq 2$ or $n \neq 1, \omega_{n}$ is an $\mathbb{F}_{p}$-module homomorphism. Let $\bar{\omega}: \lambda H \rightarrow \lambda H$ be defined by $\bar{\omega}\left(\sum_{n} a_{n}\right)=$ $\sum_{n} \omega_{n}\left(a_{n}\right)$, where $a_{n} \in \lambda_{n} H / \lambda_{n+1} H, n \geq 1$. Then, if $p \neq 2, \bar{\omega}$ is an $\mathbb{F}_{p}$-module homomorphism and, for all $p$, the restriction of $\bar{\omega}$ to $\lambda^{\prime} H$ gives an $\mathbb{F}_{p}$-module homomorphism $\bar{\omega}^{\prime}: \lambda^{\prime} H \rightarrow \lambda^{\prime} H$.

If $p \neq 2$ or $m \neq 1,\left(u^{p}, v\right) \lambda_{m+n+2} H=(u, v)^{p} \lambda_{m+n+2} H$ for all $u \in \lambda_{m} H, v \in$ $\lambda_{n} H$ : this follows from the congruence $\left(u^{p}, v\right) \equiv(u, v)^{p} \bmod \left(\gamma_{2} B\right)^{p}\left(\gamma_{p} B\right)$, where $B=\langle u,(u, v)\rangle$ (see [15, VIII.1.1b]). Thus, in this case,

$$
\left[\bar{\omega}\left(u \lambda_{m+1} H\right), v \lambda_{n+1} H\right]=\bar{\omega}\left(\left[u \lambda_{m+1} H, v \lambda_{n+1} H\right]\right) .
$$

Results (i) and (ii) follow by defining $\omega u=\bar{\omega}(u)$ for all $u \in \lambda H$.

PROPOSITION 2.2. Let $H$ be a group such that $H / \gamma_{n+1} H$ is torsion-free for all $n \geq 1$. Then there is a bijective map

$$
\alpha: \mathbb{F}_{p}[\omega] \otimes_{\mathbb{F}_{p}} \tilde{\gamma} H \rightarrow \lambda H
$$

satisfying $\alpha\left(\omega^{i} \otimes u\left(\gamma_{n} H\right)^{p} \gamma_{n+1} H\right)=u^{p^{i}} \lambda_{n+i+1} H$ for all $u \in \gamma_{n} H, i \geq 0, n \geq 1$, such that (i) for $p \neq 2, \alpha$ is an $\mathbb{F}_{p}[\omega]$-Lie algebra isomorphism, and (ii) for all $p, \alpha$ restricts to an $\mathbb{F}_{p}[\omega]$-Lie algebra isomorphism $\alpha^{\prime}: \mathbb{F}_{p}[\omega] \otimes \tilde{\gamma}^{\prime} H \rightarrow \lambda^{\prime} H$.

PROOF. Note firstly that $\mathbb{F}_{p}[\omega] \otimes \tilde{\gamma} H$ is a direct sum of $\mathbb{F}_{p}$-submodules $\omega^{i} \otimes\left(\gamma_{n} H /\left(\gamma_{n} H\right)^{p} \gamma_{n+1} H\right), i \geq 0, n \geq 1$. For $s \geq 1$ let

$$
A_{s}=\bigoplus_{n=1}^{s} \omega^{s-n} \otimes\left(\gamma_{n} H /\left(\gamma_{n} H\right)^{p} \gamma_{n+1} H\right)
$$

Thus $\mathbb{F}_{p}[\omega] \otimes \tilde{\gamma} H=\bigoplus_{s \geq 1} A_{s}$. By [15, VIII.1.9b], for each $s \geq 1$, there is a bijective map $\alpha_{s}: A_{s} \rightarrow \lambda_{s} H / \lambda_{s+1} H$ defined by

$\alpha_{s}\left(\left(\omega^{s-1} \otimes \bar{u}_{1}\right)+\left(\omega^{s-2} \otimes \bar{u}_{2}\right)+\cdots+\left(\omega^{0} \otimes \bar{u}_{s}\right)\right)=\left(u_{1}^{p^{s-1}} u_{2}^{p^{s-2}} \ldots u_{s}\right) \lambda_{s+1} H$,

where $u_{n} \in \gamma_{n} H$ and $\bar{u}_{n}=u_{n}\left(\gamma_{n} H\right)^{p} \gamma_{n+1} H(1 \leq n \leq s)$. Accordingly, let $\alpha: \mathbb{F}_{p}[\omega] \otimes \tilde{\gamma} H \rightarrow \lambda H$ be defined by $\alpha\left(\sum_{s} a_{s}\right)=\sum_{s} \alpha_{s}\left(a_{s}\right)$, where $a_{s} \in A_{s}$, $s \geq 1$. Thus $\alpha$ is bijective and

$$
\alpha\left(\omega^{i} \otimes u\left(\gamma_{n} H\right)^{p} \gamma_{n+1} H\right)=u^{p^{i}} \lambda_{n+i+1} H
$$


for all $u \in \gamma_{n} H, i \geq 0, n \geq 1$. By [15, VIII.1.9c], for $p \neq 2, \alpha$ is an $\mathbb{F}_{p^{-}}$ module isomorphism, and, for all $p, \alpha$ restricts to an $\mathbb{F}_{p}$-module isomorphism $\alpha^{\prime}: \mathbb{F}_{p}[\omega] \otimes \tilde{\gamma}^{\prime} H \rightarrow \lambda^{\prime} H$.

For all $u \in \gamma_{n} H, n \geq 1$, and all $i \geq 0$,

$$
\begin{aligned}
\alpha\left(\omega^{i+1} \otimes u\left(\gamma_{n} H\right)^{p} \gamma_{n+1} H\right) & =u^{p^{i+1}} \lambda_{n+i+2} H=\omega\left(u^{p^{i}} \lambda_{n+i+1} H\right) \\
& =\omega \alpha\left(\omega^{i} \otimes u\left(\gamma_{n} H\right)^{p} \gamma_{n+1} H\right) .
\end{aligned}
$$

It follows that, for $p \neq 2, \alpha$ is an $\mathbb{F}_{p}[\omega]$-module isomorphism, and, for all $p, \alpha^{\prime}$ is an $\mathbb{F}_{p}[\omega]$-module isomorphism. Now $\alpha\left(1 \otimes u\left(\gamma_{n} H\right)^{p} \gamma_{n+1} H\right)=u \lambda_{n+1} H$ for all $u \in \gamma_{n} H, n \geq 1$. Thus it is easily verified that $\alpha$ restricted to $1 \otimes \tilde{\gamma} H$ is a homomorphism of $\mathbb{F}_{p}$-Lie algebras. Results (i) and (ii) follow because $1 \otimes \tilde{\gamma} H$ spans $\mathbb{F}_{p}[\omega] \otimes \tilde{\gamma} H$ as $\mathbb{F}_{p}[\omega]$-module and $1 \otimes \tilde{\gamma}^{\prime} H$ spans $\mathbb{F}_{p}[\omega] \otimes \tilde{\gamma}^{\prime} H$.

For any group $H$ we write $\operatorname{End}(H)$ for the set of endomorphisms of $H$. If $\beta \in \operatorname{End}(H)$ then, since the subgroups $\gamma_{n} H$ and $\lambda_{n} H$ are fully invariant, $\beta$ induces endomorphisms of $\gamma_{n} H / \gamma_{n+1} H, \gamma_{n} H /\left(\gamma_{n} H\right)^{p} \gamma_{n+1} H$ and $\lambda_{n} H / \lambda_{n+1} H$, which we denote by $\bar{\gamma}_{n} \beta, \tilde{\gamma}_{n} \beta$ and $\bar{\lambda}_{n} \beta$, respectively. Recall that $H$ is 'relatively free' if $H \cong F / V(F)$ for some free group $F$ and some fully invariant subgroup $V(F)$ of $F$.

LEMMA 2.3. (i) Let $H$ be a group and let $\beta, \beta^{\prime} \in \operatorname{End}(H)$. If $\bar{\gamma}_{1} \beta=\bar{\gamma}_{1} \beta^{\prime}$ then $\bar{\gamma}_{n} \beta=\bar{\gamma}_{n} \beta^{\prime}$ for all $n \geq 1$. If $\bar{\lambda}_{1} \beta=\bar{\lambda}_{1} \beta^{\prime}$ then $\tilde{\gamma}_{n} \beta=\tilde{\gamma}_{n} \beta^{\prime}$ and $\bar{\lambda}_{n} \beta=\bar{\lambda}_{n} \beta^{\prime}$ for all $n \geq 1$.

(ii) If $N$ is a normal subgroup of a relatively free group $H$ and $\theta \in$ $\operatorname{End}(H / N)$ then there exists $\beta \in \operatorname{End}(H)$ such that $\beta(N) \subseteq N$ and $\beta(h) N=$ $\theta(h N)$ for all $h \in H$.

(iii) Let $H$ be a relatively free group and let $\beta \in \operatorname{End}(H)$. If $\bar{\gamma}_{1} \beta$ is an automorphism then so is $\bar{\gamma}_{n} \beta$ for all $n \geq 1$. If $\bar{\lambda}_{1} \beta$ is an automorphism then so are $\tilde{\gamma}_{n} \beta$ and $\bar{\lambda}_{n} \beta$ for all $n \geq 1$.

PROOF. The proofs of VIII.1.7a, VIII.13.3a and VIII.13.3b of [15] apply with only minor modifications.

PROPOSITION 2.4. Let $H$ be a relatively free group and write $U=H / H^{\prime}=$ $H / \gamma_{2} H$. The action of $\mathrm{GL}_{\mathbb{Z}}(U)$ on $U$ extends to $\gamma H$ so that $\gamma H$ is a $\mathbb{Z} \mathrm{GL}(U)$ module on which every element of $\mathrm{GL}(U)$ acts as a Lie ring automorphism. 
ProOF. Let $g \in \mathrm{GL}(U)$. By Lemma 2.3 (ii) there exists $\beta_{g} \in \operatorname{End}(H)$ such that $\beta_{g}$ induces $g$ on $U$. Lemma 2.3 (i), (iii) show that, for all $n \geq 1, \bar{\gamma}_{n} \beta_{g}$ is an automorphism of $\gamma_{n} H / \gamma_{n+1} H$ depending only on $g$ and not on the choice of $\beta_{g}$. The action of $g$ on $\gamma_{n} H / \gamma_{n+1} H$ is defined by $g\left(u \gamma_{n+1} H\right)=\beta_{g}(u) \gamma_{n+1} H$ for all $u \in \gamma_{n} H$, and the rest of the proof is straightforward.

A similar proof gives the analogous results for $\tilde{\gamma} H$ and $\lambda H$.

PROPOSITION 2.5. Let $H$ be a relatively free group and write $V=H / H^{p} \gamma_{2} H$ $=H / \lambda_{2} H$. The action of $\mathrm{GL}_{\mathbb{F}_{p}}(V)$ on $V$ extends to $\tilde{\gamma} H$ and $\lambda H$ so that $\tilde{\gamma} H$ and $\lambda H$ are $\mathbb{F}_{p} \mathrm{GL}(V)$-modules on which every element of $\mathrm{GL}(V)$ acts as a Lie algebra automorphism.

In the case of $\lambda H$ we can do rather better.

PROPOSITION 2.6. Let $H$ be a relatively free group and write $V=H / \lambda_{2} H$. Regard $\lambda H$ as a GL(V)-module as in Proposition 2.5. Then (i) for $p \neq 2, \lambda H$ is an $\mathbb{F}_{p}[\omega] \mathrm{GL}(V)$-module in which every element of $\mathrm{GL}(V)$ acts as an $\mathbb{F}_{p}[\omega]$-Lie algebra automorphism, and (ii) for all p, a similar statement holds for $\lambda^{\prime} H$.

PROOF. It is easily verified that $\lambda^{\prime} H$ is an $\mathbb{F}_{p} \mathrm{GL}(V)$-submodule of $\lambda H$. Let $g \in \mathrm{GL}(V)$ and let $\beta_{g}$ be an endomorphism of $H$ which induces $g$ on $V$. Let $\bar{\omega}$ be as in the proof of Proposition 2.1. Then for all $u \in \lambda_{n} H, n \geq 1$,

$$
\begin{aligned}
\bar{\omega}\left(g\left(u \lambda_{n+1} H\right)\right) & =\bar{\omega}\left(\beta_{g}(u) \lambda_{n+1} H\right)=\beta_{g}(u)^{p} \lambda_{n+2} H \\
& =\beta_{g}\left(u^{p}\right) \lambda_{n+2} H=g \bar{\omega}\left(u \lambda_{n+1} H\right) .
\end{aligned}
$$

Results (i) and (ii) now follow easily.

PROPOSITION 2.7. Let $H$ be a relatively free group such that $H / \gamma_{n+1} H$ is torsion-free for all $n \geq 1$, and write $V=H / H^{p} \gamma_{2} H=H / \lambda_{2} H$. Then the $\mathbb{F}_{p}[\omega]$-Lie algebra isomorphisms

$$
\alpha: \mathbb{F}_{p}[\omega] \otimes \tilde{\gamma} H \rightarrow \lambda H \quad(p \neq 2), \quad \alpha^{\prime}: \mathbb{F}_{p}[\omega] \otimes \tilde{\gamma}^{\prime} H \rightarrow \lambda^{\prime} H,
$$

of Proposition 2.2 are $\mathbb{F}_{p}[\omega] \mathrm{GL}(V)$-module isomorphisms.

Proof. Let $g \in \mathrm{GL}(V)$ and let $\beta_{g}$ be an endomorphism of $H$ which induces $g$ on $V$. Let $u \in \gamma_{n} H, n \geq 1$, and write $\bar{u}=u\left(\gamma_{n} H\right)^{p} \gamma_{n+1} H$. Then

$$
\begin{aligned}
\alpha(g(1 \otimes \bar{u})) & =\alpha\left(1 \otimes \beta_{g}(u)\left(\gamma_{n} H\right)^{p} \gamma_{n+1} H\right)=\beta_{g}(u) \lambda_{n+1} H \\
& =g\left(u \lambda_{n+1} H\right)=g(\alpha(1 \otimes \bar{u})) .
\end{aligned}
$$


The results follow because $1 \otimes \tilde{\gamma} H$ spans $\mathbb{F}_{p}[\omega] \otimes \tilde{\gamma} H$ as $\mathbb{F}_{p}[\omega]$-module and $1 \otimes \tilde{\gamma}^{\prime} H$ spans $\mathbb{F}_{p}[\omega] \otimes \tilde{\gamma}^{\prime} H$.

Let $E$ be the free metabelian group on a set $X$ and let $M^{\mathbb{Z}}(X)$ be the free metabelian Lie ring on $X$ (as in Section 1). By Theorem 3.2 of Shmel'kin [19] there is an isomorphism of Lie rings $\xi_{0}: M^{\mathbb{Z}}(X) \rightarrow \gamma E$ such that $\xi_{0}(x)=x \gamma_{2} E$ for all $x \in X$. Let $U=E / E^{\prime}=E / \gamma_{2} E$. Then we can use $\xi_{0}^{-1}$ to identify $U$ with the $\mathbb{Z}$-submodule of $M^{\mathbb{Z}}(X)$ spanned by $X$. Thus $M^{\mathbb{Z}}(X)=\mathbf{M} U$. It is easily verified that $\xi_{0}\left(\mathbf{M}_{n} U\right)=\gamma_{n} E / \gamma_{n+1} E$ for all $n \geq 1$. As explained in Section 1, $\mathbf{M} U$ has the structure of a $\mathbb{Z} \mathrm{GL}(U)$-module. Also (see Proposition 2.4), $\gamma E$ has the structure of a $\mathbb{Z G L}(U)$-module. For all $u \in U$ and all $g \in \mathrm{GL}(U)$ we have $\xi_{0}(g u)=g u=g \xi_{0}(u)$ because of the identification of $U$. Since $U$ generates $\mathbf{M} U$ as a Lie ring, and $\mathrm{GL}(U)$ acts by Lie ring automorphisms on $\mathbf{M} U$ and $\gamma E$, it follows that $\xi_{0}(g u)=g \xi_{0}(u)$ for all $u \in \mathbf{M} U$. Thus $\xi_{0}$ is an isomorphism of $\mathbb{Z} \mathrm{GL}(U)$-modules and we have proved the following result.

PROPOSITION 2.8. Let $E$ be a free metabelian group and write $U=E / E^{\prime}=$ $E / \gamma_{2} E$. There is a bijective map $\xi_{0}: \mathbf{M} U \rightarrow \gamma E$ which is an isomorphism of Lie rings and of $\mathbb{Z} \mathrm{GL}(U)$-modules such that $\xi\left(\mathbf{M}_{n} U\right)=\gamma_{n} E / \gamma_{n+1} E$ for all $n \geq 1$.

Now $M^{\mathbb{F}_{p}}(X) \cong \mathbb{F}_{p} \otimes_{\mathbb{Z}} M^{\mathbb{Z}}(X)$ and $\tilde{\gamma} E \cong \mathbb{F}_{p} \otimes_{\mathbb{Z}} \gamma E$. Thus $\xi_{0}$ gives an $\mathbb{F}_{p}$-Lie algebra isomorphism $\xi: M^{\mathbb{F}_{p}}(X) \rightarrow \tilde{\gamma} E$. We can repeat the proof of Proposition 2.8 to obtain an analogous result for $\tilde{\gamma} E$.

PROPOSITION 2.9. Let $E$ be a free metabelian group and write $V=E / E^{p} \gamma_{2} E$ $=E / \lambda_{2} E$. There is a bijective map $\xi: \mathbf{M} V \rightarrow \tilde{\gamma} E$ which is an isomorphism of $\mathbb{F}_{p}$-Lie algebras and of $\mathbb{F}_{p} \mathrm{GL}(V)$-modules such that $\xi\left(\mathbf{M}_{n} V\right)=$ $\gamma_{n} E /\left(\gamma_{n} E\right)^{p} \gamma_{n+1} E$ for all $n \geq 1$.

We can now prove the main result of this section.

PROPOSITION 2.10. Let $E$ be a free metabelian group and write $V=E / \lambda_{2} E$.

(i) For $p \neq 2$, and $s \geq 1$, there is an $\mathbb{F}_{p} \mathrm{GL}(V)$-module isomorphism

$$
\lambda_{s} E / \lambda_{s+1} E \cong \bigoplus_{n=1}^{s} \mathbf{M}_{n} V .
$$

(ii) For all $p$, and $s \geq 2$, there is an $\mathbb{F}_{p} \mathrm{GL}(V)$-module isomorphism

$$
\left(E^{\prime} \cap \lambda_{s} E\right) \lambda_{s+1} E / \lambda_{s+1} E \cong \bigoplus_{n=2}^{s} \mathbf{M}_{n} V .
$$


PROOF. Note first that Proposition 2.7 is applicable with $H=E$ : the fact that $E / \gamma_{n+1} E$ is torsion-free for all $n \geq 1$ is contained in the work of Chen [9] although probably proved earlier by Magnus (see [18, 36.32]).

(i) By Proposition 2.7 the map $\alpha^{-1}: \lambda E \rightarrow \mathbb{F}_{p}[\omega] \otimes \tilde{\gamma} E$ is an $\mathbb{F}_{p}[\omega] \mathrm{GL}(V)$ module isomorphism, hence an $\mathbb{F}_{p} \mathrm{GL}(V)$-module isomorphism. But

$$
\begin{aligned}
\alpha^{-1}\left(\lambda_{s} E / \lambda_{s+1} E\right) & =\bigoplus_{n=1}^{s} \omega^{s-n} \otimes\left(\gamma_{n} E /\left(\gamma_{n} E\right)^{p} \gamma_{n+1} E\right) \\
& \cong \bigoplus_{n=1}^{s} \gamma_{n} E /\left(\gamma_{n} E\right)^{p} \gamma_{n+1} E
\end{aligned}
$$

as $\mathbb{F}_{p} \mathrm{GL}(V)$-module. The result follows by Proposition 2.9.

(ii) Similarly, using $\alpha^{\prime}$, we obtain $\mathbb{F}_{p} \mathrm{GL}(V)$-module isomorphisms

$$
\begin{aligned}
\left(E^{\prime} \cap \lambda_{s} E\right) \lambda_{s+1} E / \lambda_{s+1} E & \cong \bigoplus_{n=2}^{s} \omega^{s-n} \otimes\left(\gamma_{n} E /\left(\gamma_{n} E\right)^{p} \gamma_{n+1} E\right) \\
& \cong \bigoplus_{n=2}^{s} \gamma_{n} E /\left(\gamma_{n} E\right)^{p} \gamma_{n+1} E .
\end{aligned}
$$

The result follows by Proposition 2.9 .

REMARK. Similar proofs give analogues of Propositions 2.8, 2.9 and 2.10 for a free group $F$, describing $\gamma F, \tilde{\gamma} F$ and $\lambda F$ in terms of the free Lie algebras $\mathbf{L}\left(F / \gamma_{2} F\right)$ and $\mathbf{L}\left(F / \lambda_{2} F\right)$. Similarly (using results from [19]) we can obtain analogues for any free polynilpotent group $H$, describing $\gamma H, \tilde{\gamma} H$ and $\lambda H$ in terms of associated free polynilpotent Lie algebras $\mathbf{P}\left(H / \gamma_{2} H\right)$ and $\mathbf{P}\left(H / \lambda_{2} H\right)$.

We conclude this section by recording a fact which will be used repeatedly : in the applications $K^{\prime}$ will either be an extension ring of $K$ or a factor ring of $K$.

PROPOSITION 2.11. Let $K \rightarrow K^{\prime}$ be a homomorphism of non-zero commutative rings with identity (so that $K^{\prime}$ can be regarded as $a\left(K^{\prime}, K\right)$-bimodule). Let $H$ be any group and let $V$ be $a \mathrm{KH}$-module which is free as a $K$-module. Let $\mathrm{D}$ denote $\mathbf{S}, \mathbf{L}$ or $\mathbf{M}$. Then there is an isomorphism $K^{\prime} \otimes_{K} \mathbf{D} V \rightarrow \mathbf{D}\left(K^{\prime} \otimes_{K} V\right)$ which is an isomorphism of $K^{\prime}$-algebras and of $K^{\prime} H$-modules such that $1 \otimes v \mapsto 1 \otimes v$ for all $v \in V$.

PROOF. The result essentially follows from the universal properties of free algebras and tensor products. We omit the details (cf. $[5, \S 2,5.3]$ ). 


\section{Regular modules and automorphisms}

In this section we shall prove Theorems $\mathrm{A}$ and $\mathrm{B}$. We begin with an analogue of Theorem A for $\mathbf{S} V$. Although this result is essentially well known (see, for example, the proof of Theorem 7.1 of [1]), the simple proof given here seems worthy of note.

PROPOSITION 3.1. Let $V$ be a non-zero finite dimensional vector space over a field $K$ and let $G$ be a finite subgroup of $\mathrm{GL}(V)$, where $|G|=c$. Then, for all $n \geq 0, \mathbf{S}_{n} V \oplus \cdots \oplus \mathbf{S}_{n+c-1} V$ has a regular $K G$-submodule.

PROOF. Let $G=\left\{g_{1}, \ldots, g_{c}\right\}$. By Proposition 2.11 and [15, VII.7.23], we may assume that $K$ is infinite (by passing to an infinite extension field of $K$ if necessary). For each $g \in G \backslash\{1\},\{v \in V: g v=v\}$ is a proper subspace of $V$. Hence, since $K$ is infinite, there exists a non-zero element $v$ of $V$ such that $g v \neq v$ for all $g \in G \backslash\{1\}$; thus the elements $g_{1} v, \ldots, g_{c} v$ are distinct. Hence, in the integral domain $\mathbf{S} V$, the van der Monde determinant

$$
\left|\begin{array}{lll}
\left(g_{1} v\right)^{n} & \ldots & \left(g_{c} v\right)^{n} \\
\left(g_{1} v\right)^{n+1} & \ldots & \left(g_{c} v\right)^{n+1} \\
\vdots & \ddots & \vdots \\
\left(g_{1} v\right)^{n+c-1} & \ldots & \left(g_{c} v\right)^{n+c-1}
\end{array}\right|
$$

is non-zero. Hence the columns of the van der Monde matrix are linearly independent regarded as vectors over the field of fractions of $\mathbf{S} V$. Therefore they are linearly independent over $K$. By comparing the homogeneous components, it follows that the elements $\left(g_{i} v\right)^{n}+\left(g_{i} v\right)^{n+1}+\cdots+\left(g_{i} v\right)^{n+c-1}$ of $\mathbf{S} V(1 \leq i \leq c)$ are linearly independent over $K$. Let $u=v^{n}+v^{n+1}+\cdots+v^{n+c-1}$. Then, for each $i, g_{i} u=\left(g_{i} v\right)^{n}+\cdots+\left(g_{i} v\right)^{n+c-1}$. Hence the elements $g_{1} u, \ldots, g_{c} u$ are linearly independent over $K$. It follows that $u$ generates a regular $K G$-submodule of $\mathbf{S}_{n} V+\cdots+\mathbf{S}_{n+c-1} V$.

REMARK. From Proposition 3.1 we can easily deduce the corresponding result for $\mathbf{A} V$, the free associative algebra or tensor algebra on $V$. This is because $\mathbf{S} V$ is isomorphic to a factor algebra $\mathbf{A} V / I$, and if $u+I$ is an element of $\mathbf{A} V / I$ which generates a regular $K G$-module then $u$ generates a regular $K G$-submodule of $\mathbf{A} V$. Thus we obtain a simple proof of a generalisation of the well known result of Burnside concerned with the occurrence of irreducible modules in tensor powers. (For further background see [7].) 
Let $V$ be a vector space over a field $K$ with finite dimension $d \geq 2$ and basis $X=\left\{x_{1}, \ldots, x_{d}\right\}$. We temporarily use the notation $S_{(1)}=\bigoplus_{j \geq 1} \mathbf{s}_{j} V$ and $M_{(2)}=\bigoplus_{j \geq 2} \mathbf{M}_{j} V$. Every element of $S_{(1)}$ can be written uniquely in the form

$$
w=\sum \rho_{\left(n_{1}, \ldots, n_{d}\right)} x_{1}^{n_{1}} \ldots x_{d}^{n_{d}}
$$

where the sum is over all ordered $d$-tuples $\left(n_{1}, \ldots, n_{d}\right)$ of non-negative integers not all equal to 0 and where the $\rho_{\left(n_{1}, \ldots, n_{d}\right)}$ are elements of $K$ all but finitely many of which are 0 . If $u \in M_{(2)}$ and $w$ is as given by (1) we write $[u ; w]$ for the element of $M_{(2)}$ given by

$$
\begin{array}{r}
{[u ; w]=\sum \rho_{\left(n_{1}, \ldots, n_{d}\right)}\left[u, x_{1}, \ldots, x_{1}, \ldots, x_{d}, \ldots, x_{d}\right]} \\
\leftarrow n_{1} \rightarrow \underset{n_{d}}{\leftarrow} \rightarrow
\end{array}
$$

where, in the term corresponding to $\left(n_{1}, \ldots, n_{d}\right)$, there are $n_{i}$ occurrences of $x_{i}$ for each $i$. Since $\mathbf{M} V$ is metabelian,

$$
\left[u, x_{i}, x_{j}\right]=\left[u, x_{j}, x_{i}\right]
$$

for all $u \in M_{(2)}$ and all $i, j \in\{1, \ldots, d\}$. Hence, for all $u \in M_{(2)}$ and $w_{1}$, $w_{2} \in S_{(1)}$,

$$
\left[u ; w_{1} w_{2}\right]=\left[\left[u ; w_{1}\right] ; w_{2}\right]
$$

Also

$$
\left[u ; w_{1}+w_{2}\right]=\left[u ; w_{1}\right]+\left[u ; w_{2}\right]
$$

For all $g \in \mathrm{GL}(V), u \in M_{(2)}, v \in V=\mathbf{S}_{1} V$, we have

$$
g[u ; v]=g[u, v]=[g u, g v]=[g u ; g v] .
$$

Hence, by (3), (4) and induction,

$$
g[u ; w]=[g u ; g w]
$$

for all $g \in \mathrm{GL}(V), u \in M_{(2)}, w \in S_{(1)}$. The tensor product $\mathbf{M}_{2} V \otimes_{K} S_{(1)}$ has the structure of a $K \mathrm{GL}(V)$-module under the 'diagonal' action of $\mathrm{GL}(V)$ : $g(u \otimes w)=g u \otimes g w$ for all $g \in \mathrm{GL}(V), u \in \mathbf{M}_{2} V, w \in S_{(1)}$. From (5) we obtain the following result. 
LEMMA 3.2. There is a $\mathrm{KGL}(V)$-module homomorphism

$$
\eta: \mathbf{M}_{2} V \otimes_{K}\left(\bigoplus_{j \geq 1} \mathbf{S}_{j} V\right) \rightarrow \mathbf{M} V
$$

such that $\eta(u \otimes w)=[u ; w]$ for all $u \in \mathbf{M}_{2} V, w \in \bigoplus_{j \geq 1} \mathbf{S}_{j} V$.

We shall first prove Theorem A in the case where $K$ has non-zero characteristic.

PROPOSITION 3.3. Let $V$ be a finite dimensional vector space of dimension at least 2 over a field $K$ of characteristic $p>0$ and let $G$ be a finite subgroup of $\mathrm{GL}(V)$. For all $n \geq 1$ there exists $t \geq n$ such that $\mathbf{M}_{n} V \oplus \cdots \oplus \mathbf{M}_{t} V$ has $a$ regular $K G$-submodule.

Proof. As before, let $\left\{x_{1}, \ldots, x_{d}\right\}$ be a basis of $V$ and let $G=\left\{g_{1}, \ldots, g_{c}\right\}$ where $|G|=c$. As in the proof of Proposition 3.1 we may assume that $K$ is infinite and take $v \in V$ so that the elements $g_{1} v, \ldots, g_{c} v$ are distinct. Let $n \geq 1$ and take a positive integer $m$ so that $p^{m} \geq n$. Since $\mathbf{S} V$ has characteristic $p$,

$$
\left(g_{i} v\right)^{p^{m}}-\left(g_{j} v\right)^{p^{m}}=\left(g_{i} v-g_{j} v\right)^{p^{m}} \neq 0
$$

for all $i, j(i \neq j)$. Thus the argument used in the proof of Proposition 3.1 shows that the element

$$
v^{p^{m}}+v^{2 p^{m}}+\cdots+v^{c p^{m}}
$$

generates a regular $K G$-submodule $W$ of $\mathbf{S} V$.

Let $Z$ be the subalgebra of $\mathbf{S} V$ generated by $x_{1}^{p}, \ldots, x_{d}^{p}$. Clearly $Z$ is a $K G$-submodule of $\mathbf{S} V$ and $W \subseteq Z \subseteq \bigoplus_{j \geq 1} \mathbf{S}_{j} V$. Let $\eta$ be the $K G$-module homomorphism given by Lemma 3.2. Then

$$
\eta\left(\mathbf{M}_{2} V \otimes W\right) \subseteq \bigoplus_{n \leq j \leq t} \mathbf{M}_{j} V
$$

for some $t$, and $\mathbf{M}_{2} V \otimes W$ is a free $K G$-module by [15, VII.7.19a]. Thus to prove the proposition it is sufficient to prove that $\eta$ restricted to $\mathbf{M}_{2} V \otimes W$ is injective. We do this by showing that $\eta$ restricted to $\mathbf{M}_{2} V \otimes Z$ is injective.

Now

$$
Z=\bigoplus_{\substack{k \geq 1 \\ p \mid k}} Z \cap \mathbf{S}_{k} V
$$


and, for each $k$,

$$
\eta\left(\mathbf{M}_{2} V \otimes\left(Z \cap \mathbf{S}_{k} V\right)\right) \subseteq \mathbf{M}_{k+2} V .
$$

Thus it is sufficient to prove that $\eta$ restricted to $\mathbf{M}_{2} V \otimes\left(Z \cap \mathbf{S}_{k} V\right)$ is injective for each $k$.

Let $A$ be the set of all elements of $\mathbf{S} V$ of the form $x_{1}^{\beta_{1}} \ldots x_{d}^{\beta_{d}}$ where $\beta_{1}+$ $\cdots+\beta_{d}=k$ and all of $\beta_{1}, \ldots, \beta_{d}$ are divisible by $p$. Then $A$ is a $K$-basis of $Z \cap \mathbf{S}_{k} V$. Furthermore, the sets

$$
B_{2}=\left\{\left[x_{i_{1}}, x_{i_{2}}\right]: i_{1}>i_{2}\right\}
$$

and

$$
B_{k+2}=\left\{\left[x_{i_{1}}, \ldots, x_{i_{k+2}}\right]: i_{1}>i_{2} \leq i_{3} \leq \ldots \leq i_{k+2}\right\}
$$

are $K$-bases of $\mathbf{M}_{2} V$ and $\mathbf{M}_{k+2} V$, respectively (see [2, 15.3.2]). For each $d$-tuple $\alpha=\left(\alpha_{1}, \ldots, \alpha_{d}\right)$ of non-negative integers satisfying $\alpha_{1}+\cdots+\alpha_{d}=k+2$ let $N_{\alpha}$ be the $K$-subspace of $\mathbf{M}_{k+2} V$ spanned by all those basis elements $\left[x_{i_{1}}, \ldots, x_{i_{k+2}}\right]$ of $B_{k+2}$ for which there are exactly $\alpha_{j}$ of the subscripts $i_{1}, \ldots, i_{k+2}$ equal to $j$ $(j=1, \ldots, d)$. Thus $\mathbf{M}_{k+2} V$ is the direct sum of the subspaces $N_{\alpha}$.

The elements $b \otimes a\left(b \in B_{2}, a \in A\right)$ form a basis for $\mathbf{M}_{2} V \otimes\left(Z \cap \mathbf{S}_{k} V\right)$. It is sufficient to show that the elements $\eta(b \otimes a)$ are linearly independent. Thus it is sufficient to show that, for each $b \otimes a, \eta(b \otimes a)$ is a non-zero element of $N_{\alpha}$ for some $\alpha=\alpha(b \otimes a)$ depending on $b \otimes a$, and that $\alpha(b \otimes a) \neq \alpha\left(b^{\prime} \otimes a^{\prime}\right)$ for distinct basis elements $b \otimes a$ and $b^{\prime} \otimes a^{\prime}$.

Let $b=\left[x_{i_{1}}, x_{i_{2}}\right]$ and $a=x_{1}^{\beta_{1}} \ldots x_{d}^{\beta_{d}}$. Write $\alpha=\left(\alpha_{1}, \ldots, \alpha_{d}\right)$ where $\alpha_{i_{1}}=$ $\beta_{i_{1}}+1, \alpha_{i_{2}}=\beta_{i_{2}}+1$ and $\alpha_{j}=\beta_{j}\left(j \neq i_{1}, i_{2}\right)$. Let $s$ be the least integer for which $\beta_{s} \neq 0$. Thus

$$
\eta(b \otimes a)=\left[\left[x_{i_{1}}, x_{i_{2}}\right] ; x_{s}^{\beta_{s}} \ldots x_{d}^{\beta_{d}}\right]=\left[x_{i_{1}}, x_{i_{2}}, x_{s}, \ldots, x_{s}, \ldots, x_{d}, \ldots, x_{d}\right] .
$$

If $i_{2} \leq s$ then $\eta(b \otimes a)$ is an element of $B_{k+2}$ and so $\eta(b \otimes a) \neq 0$. Clearly $\eta(b \otimes a) \in N_{\alpha}$ in this case. If $i_{2}>s$ then, by the Jacobi identity and (2), we can write $\eta(b \otimes a)$ in the form

$$
-\left[x_{i_{2}}, x_{s}, \ldots, x_{s}, \ldots, x_{i_{1}}, \ldots\right]+\left[x_{i_{1}}, x_{s}, \ldots, x_{s}, \ldots, x_{i_{2}}, \ldots\right],
$$

the difference of two distinct elements of $B_{k+2}$. Thus $\eta(b \otimes a) \neq 0$ and $\eta(b \otimes a) \in N_{\alpha}$ in this case also.

It remains to prove that if $b \otimes a$ and $b^{\prime} \otimes a^{\prime}$ are basis elements such that $\alpha(b \otimes a)=\alpha\left(b^{\prime} \otimes a^{\prime}\right)$ then $b \otimes a=b^{\prime} \otimes a^{\prime}$. In other words we have to show that $\alpha(b \otimes a)$ determines $b \otimes a$ uniquely. Suppose that $b=\left[x_{i_{1}}, x_{i_{2}}\right], a=x_{1}^{\beta_{1}} \ldots x_{d}^{\beta_{d}}$ 
and $\alpha(b \otimes a)=\left(\alpha_{1}, \ldots, \alpha_{d}\right)$, as before. Then $\alpha_{i_{1}}=\beta_{i_{1}}+1, \alpha_{i_{2}}=\beta_{i_{2}}+1$ and $\alpha_{j}=\beta_{j}\left(j \neq i_{1}, i_{2}\right)$. Since each $\beta_{i}$ is divisible by $p,\left(\alpha_{1}, \ldots, \alpha_{d}\right)$ determines $i_{1}$ and $i_{2}$. Thus $\left(\alpha_{1}, \ldots, \alpha_{d}\right)$ determines $\left(\beta_{1}, \ldots, \beta_{d}\right)$. Hence $a$ and $b$ are uniquely determined by $\alpha(b \otimes a)$.

We can now deal with the characteristic 0 case of Theorem $A$. This is obtained from the prime characteristic case by the standard process of modular reduction.

PROPOSITION 3.4. Let $V$ be a finite dimensional vector space of dimension at least 2 over a field $K$ of characteristic 0 and let $G$ be a finite subgroup of $\mathrm{GL}(V)$. For all $n \geq 1$ there exists $t \geq n$ such that $\mathbf{M}_{n} V \oplus \cdots \oplus \mathbf{M}_{t} V$ has $a$ regular $K G$-submodule.

PROOF. By Proposition 2.11 and [15, VII.7.23] we may assume that $K$ is an algebraically closed extension field of the rational field $\mathbb{Q}$. By [10, (1.11)] there exists a subfield $\mathscr{F}$ of $K$ such that $\mathscr{F}$ is finite dimensional over $\mathbb{Q}$ and $\mathscr{F}$ is a splitting field for $G$. Hence there is a basis of $V$ such that the matrices representing $G$ with respect to this basis have their entries in $\mathscr{F}$. Since $K G \cong$ $K \otimes_{\mathscr{F}} \mathscr{F} G$ it is enough to prove the result with $K$ replaced by $\mathscr{F}$.

Let $p$ be a prime such that $p \nmid|G|$ and let $\mathfrak{p}_{0}$ be a prime ideal in the ring of algebraic integers of $\mathscr{F}$ such that $p \in \mathfrak{p}_{0}$. Let $\mathscr{D}$ be the subring of $\mathscr{F}$ consisting of all elements $a / b$ where $a$ and $b$ are integers of $\mathscr{F}$ such that $b \notin \mathfrak{p}_{0}$. As shown in [10, pp. 24-25], $\mathscr{D}$ has a unique maximal ideal and the factor ring by this ideal is a finite field $\mathscr{D}$ of characteristic $p$. Let us say that $\mathscr{D} G$-modules $I$ and $J$ are $\mathscr{F} G$-equivalent if the $\mathscr{F} G$-modules $\mathscr{F} \otimes_{\mathscr{D}} I$ and $\mathscr{F} \otimes_{\mathscr{D}} J$ are isomorphic. For any $\mathscr{D} G$-module $I$, let $\bar{I}$ denote the corresponding $\overline{\mathscr{D}} G$-module: $\bar{I}=\overline{\mathscr{D}} \otimes_{\mathscr{D}} I$. By $[10,(4.4)]$ the mapping $I \mapsto \bar{I}$ yields a bijective mapping from the set of $\mathscr{F} G$-equivalence classes of finitely generated $\mathscr{D} G$-modules which are free as $\mathscr{D}$-modules to the set of isomorphism classes of finitely generated $\overline{\mathscr{D}} G$-modules. It follows (by considering the same correspondence for a proper factor group of $G$ ) that if $I$ is faithful as a $G$-module then so is $\bar{I}$. Clearly $\overline{\mathscr{D} G} \cong \overline{\mathscr{D}} G$. Thus if $\bar{I}$ has a regular submodule we can write $\bar{I} \cong \overline{\mathscr{D} G} \oplus \bar{J} \cong \overline{\mathscr{D} G \oplus J}$ for some $\mathscr{D} G$-module $J$ which is free as $\mathscr{D}$-module, and so $I$ is $\mathscr{F} G$-equivalent to $\mathscr{D} G \oplus J$.

By $[10,(4.1)]$ there is an $\mathscr{F}$-basis $Y$ of $V$ such that the matrices representing $G$ with respect to $Y$ have their entries in $\mathscr{D}$. Let $V_{0}$ be the (free) $\mathscr{D}$-module spanned by $Y$. Thus $\mathbf{M} V \cong \mathscr{F} \otimes_{\mathscr{D}} \mathbf{M} V_{0}$. By the remarks above $\bar{V}_{0}$ is faithful as a $G$ module. Thus we may regard $G$ as a subgroup of $\operatorname{GL}\left(\bar{V}_{0}\right)$. By Proposition 2.11, 
$\overline{\mathscr{D}} \otimes_{\mathscr{D}} \mathbf{M} V_{0} \cong \mathbf{M} \bar{V}_{0}$ and, by Proposition 3.3, there exists $t \geq n$ such that $\mathbf{M}_{n} \bar{V}_{0} \oplus \cdots \oplus \mathbf{M}_{t} \bar{V}_{0}$ has a regular $\overline{\mathscr{D}} G$-submodule. Let $I=\mathbf{M}_{n} V_{0} \oplus \cdots \oplus \mathbf{M}_{t} V_{0}$ and regard $I$ as a $\mathscr{D} G$-module. Then $\bar{I} \cong \mathbf{M}_{n} \bar{V}_{0} \oplus \cdots \oplus \mathbf{M}_{t} \bar{V}_{0}$, so $\bar{I}$ has a regular $\overline{\mathscr{D}} G$-submodule. By the remarks above it follows that $\mathscr{F} \otimes_{\mathscr{D}} I$ has a regular $\mathscr{F} G$-submodule. In other words, $\mathbf{M}_{n} V \oplus \cdots \oplus \mathbf{M}_{t} V$ has a regular $\mathscr{F} G$-submodule, as required.

PROOF OF THEOREM B. Let $p$ be a prime number and let $G$ be a linear group of finite dimension $d \geq 2$ over $\mathbb{F}_{p}$. Let $F$ be a free group of rank $d$ and let $E=F / F^{\prime \prime}$, a free metabelian group of rank $d$. Then $G$ can be identified as a linear group with a subgroup of $\hat{G}=\operatorname{GL}(V)$, where $V=E / \lambda_{2} E$. Note that $\hat{G}$ is a finite group. By Theorem A there exists $t \geq 2$ such that $\mathbf{M}_{2} V \oplus \cdots \oplus \mathbf{M}_{t} V$ has a regular $\mathbb{F}_{p} \hat{G}$-submodule. By Proposition $2.10, \lambda_{t} E / \lambda_{t+1} E$ has a submodule isomorphic to $\mathbf{M}_{2} V \oplus \cdots \oplus \mathbf{M}_{t} V$ and so has a regular $\mathbb{F}_{p} \hat{G}$-submodule. Let $P^{*}=E / \lambda_{t+1} E$ : thus $P^{*}$ is a finite metabelian $p$-group. Take $w$ to be an element of $\lambda_{t} E / \lambda_{t+1} E$ which generates a regular $\mathbb{F}_{p} \hat{G}$-submodule and let $W_{G}$ be the $\mathbb{F}_{p} G$-submodule generated by $w$. Then $W_{G}$ is a central subgroup of $P^{*}$ since $\lambda_{t} E / \lambda_{t+1} E$ is central in $P^{*}$. Take $P=P^{*} / W_{G}$. Then the method of proof of $[8$, Theorem 1] shows that $P$ has the required properties.

\section{Characters and higher relation modules}

Let $K$ be a field of characteristic 0 and let $V$ be a vector space over $K$ of finite dimension $d$. We shall consider the characters $\chi_{\mathbf{s}_{n} v}$ and $\chi_{\mathbf{M}_{n} V}$ of GL(V). Let $y$ be an indeterminate over $K$ and let $K[[y]]$ be the ring of formal power series in $y$ with coefficients from $K$. For $g \in \mathrm{GL}(V)$ we define $H_{g}=\sum_{n \geq 0} \chi_{\mathbf{s}_{n} V}(g) y^{n}$ and $M_{g}=\sum_{n \geq 1} \chi_{\mathbf{M}_{n} V}(g) y^{n}$. (In other words, $H_{g}$ and $M_{g}$ are the 'generating functions' of $\chi_{\mathbf{S}_{n} V(g)}$ and $\chi_{\mathbf{M}_{n} V(g)}$.) We write $\chi(g)$ instead of $\chi_{V}(g)$. Theorems C and $\mathrm{D}$ will be derived using the following result, at least part of which is well known (see, for example, the closely related formulae in [17, Ch. I, Section 2]).

PROPOSITION 4.1. (i) For all $g \in \mathrm{GL}(V)$,

$$
H_{g}=\prod_{k \geq 1}\left(1-y^{k}\right)^{e_{g}(k)}=\prod_{n \geq 1} \exp \left(\frac{1}{n} \chi\left(g^{n}\right) y^{n}\right),
$$


where

$$
e_{g}(k)=-\frac{1}{k} \sum_{i \mid k} \mu(i) \chi\left(g^{k / i}\right) .
$$

(ii) For all $g \in \mathrm{GL}(V)$,

$$
M_{g}=(\chi(g) y-1) H_{g}+\chi(g) y+1 .
$$

In the statement of Proposition 4.1, $\mu$ denotes the Möbius function and we have used the notation $(1+u)^{a}=\sum_{i \geq 0}\left(\begin{array}{c}a \\ i\end{array}\right) u^{i}$ for $u \in y K[[y]], a \in K$, where $\left(\begin{array}{l}a \\ i\end{array}\right)=a(a-1) \ldots(a-i+1) / i$ !, and $\exp (u)=\sum_{i \geq 0} u^{i} / i$ ! for $u \in y K[[y]]$.

PROOF. Choose a basis $X=\left\{x_{1}, \ldots, x_{d}\right\}$ of $V$. Thus we regard each element of GL( $V)$ as a $d \times d$ matrix $g=\left(g_{i j}\right)$. Choose bases for each $\mathbf{S}_{n} V$ and $\mathbf{M}_{n} V$. Then it is easily seen that the entries of the matrices representing $g$ on $\mathbf{S}_{n} V$ and $\mathbf{M}_{n} V$ are homogeneous polynomials over $K$ of total degree $n$ in the $g_{i j}$. In other words, $\mathbf{S}_{n} V$ and $\mathbf{M}_{n} V$ afford polynomial representations of GL( $\left.V\right)$ of degree $n$ (see $[12, \S 2.2]$ ). Thus, by $[12,(3.4 \mathrm{e})]$, there are polynomials $\sigma_{n}$ and $\tau_{n}$ in $d$ indeterminates with integer coefficients such that, for all $g \in \mathrm{GL}(V)$, $\chi_{\mathbf{S}_{n} V}(g)=\sigma_{n}\left(\varepsilon_{1}, \ldots, \varepsilon_{d}\right)$ and $\chi_{\mathbf{M}_{n} V}(g)=\tau_{n}\left(\varepsilon_{1}, \ldots, \varepsilon_{d}\right)$, where $\varepsilon_{1}, \ldots, \varepsilon_{d}$ are the eigenvalues of $g$ (in some extension field of $K$ ).

Equations (i) and (ii) can be interpreted as giving $\chi_{\mathbf{S}_{n} V}(g)$ and $\chi_{\mathbf{M}_{n} V}(g)$ as polynomials over $K$ in $\varepsilon_{1}, \ldots, \varepsilon_{d}$ (because $\left.\chi\left(g^{i}\right)=\varepsilon_{1}^{i}+\cdots+\varepsilon_{d}^{i}\right)$. These polynomials will coincide with $\sigma_{n}$ and $\tau_{n}$ if they do so for all $\varepsilon_{1}, \ldots, \varepsilon_{d} \in K \backslash\{0\}$, since $K$ is infinite. Thus it is sufficient to verify (i) and (ii) in the case where $g$ is a diagonal matrix,

$$
g=\left(\begin{array}{ccc}
\varepsilon_{1} & & 0 \\
& \ddots & \\
0 & & \varepsilon_{d}
\end{array}\right), \varepsilon_{1}, \ldots, \varepsilon_{d} \in K \backslash\{0\} .
$$

Note that $\mathbf{S}_{n} V$ has the basis $\left\{x_{i_{1}} x_{i_{2}} \ldots x_{i_{n}}: i_{1} \leq i_{2} \leq \ldots \leq i_{n}\right\}$. Thus $\chi_{\mathrm{s}_{n} V}(g)=h_{n}\left(\varepsilon_{1}, \ldots, \varepsilon_{d}\right)$ where $h_{n}$ is the $n$th complete symmetric function (see $[17$, p. 14]). Thus

$$
H_{g}=\sum_{n \geq 0} h_{n}\left(\varepsilon_{1}, \ldots, \varepsilon_{d}\right) y^{n}=\prod_{i=1}^{d}\left(1-\varepsilon_{i} y\right)^{-1}
$$

By [17, Ch. I, (2.10)] we have

$$
H_{g}^{\prime} / H_{g}=\sum_{n \geq 1} p_{n}\left(\varepsilon_{1}, \ldots, \varepsilon_{d}\right) y^{n-1}=\sum_{n \geq 1} \chi\left(g^{n}\right) y^{n-1}
$$


where $p_{n}$ is the $n$th power sum and $H_{g}^{\prime}$ is the formal derivative of $H_{g}$ with respect to $y$. For $k \geq 1$ define

$$
z_{g}(k)=\sum_{i \backslash k} \mu(i) \chi\left(g^{k / i}\right)
$$

Möbius inversion gives, for $n \geq 1$,

$$
\chi\left(g^{n}\right)=\sum_{k \mid n} z_{g}(k)
$$

Thus

$$
\begin{aligned}
H_{g}^{\prime} / H_{g} & =\sum_{n \geq 1} \sum_{k \mid n} \sum_{i \mid k} \mu(i) \chi\left(g^{k / i}\right) y^{n-1} \\
& =\sum_{\substack{i, k \geq 1 \\
i \mid k}} \mu(i) \chi\left(g^{k / i}\right) y^{k-1}\left(1-y^{k}\right)^{-1} .
\end{aligned}
$$

(i) follows by formal integration of (6) and (7).

By $[2,15.3 .2]$, for $n \geq 2, \mathbf{M}_{n} V$ has the basis

$$
\left\{\left[x_{i_{1}}, x_{i_{2}}, \ldots, x_{i_{n}}\right]: i_{1}>i_{2} \leq i_{3} \leq \ldots \leq i_{n}\right\} .
$$

Thus

$$
\begin{aligned}
\chi_{\mathbf{M}_{n} v}(g) & =\sum_{i_{1}>i_{2} \leq i_{3} \leq \ldots \leq i_{n}} \varepsilon_{i_{1}} \varepsilon_{i_{2}} \ldots \varepsilon_{i_{n}} \\
& =\sum_{i_{1}=1}^{d} \sum_{i_{2} \leq i_{3} \leq \ldots \leq i_{n}} \varepsilon_{i_{1}} \varepsilon_{i_{2}} \ldots \varepsilon_{i_{n}}-\sum_{i_{1} \leq i_{2} \leq i_{3} \leq \ldots \leq i_{n}} \varepsilon_{i_{1}} \varepsilon_{i_{2}} \ldots \varepsilon_{i_{n}} \\
& =h_{1}\left(\varepsilon_{1}, \ldots, \varepsilon_{d}\right) \\
h_{n-1}\left(\varepsilon_{1}, \ldots, \varepsilon_{d}\right)-h_{n} & \left(\varepsilon_{1}, \ldots, \varepsilon_{d}\right) .
\end{aligned}
$$

Clearly, $\chi_{\mathbf{M}_{1} v}(g)=h_{1}\left(\varepsilon_{1}, \ldots, \varepsilon_{d}\right)$. Thus we obtain

$$
M_{g}=\chi(g) y+\sum_{n \geq 2}\left(\chi(g) \chi \mathbf{s}_{n-1} v(g) y^{n}-{\chi \mathbf{s}_{n} V}(g) y^{n}\right),
$$

and (ii) follows.

A simpler version of the last proof gives the following well known formula for the dimension of $\mathbf{M}_{n} V$ (see [9, Corollary 1] for example). 
PROPOSITION 4.2. For $n \geq 2$,

$$
\operatorname{dim} \mathbf{M}_{n} V=(n-1)\left(\begin{array}{c}
d+n-2 \\
n
\end{array}\right) .
$$

PROOF. By the previous proof, the number of basis elements of $\mathbf{M}_{n} V$ is equal to $\left(\operatorname{dim} \mathbf{S}_{1} V\right)\left(\operatorname{dim} \mathbf{S}_{n-1} V\right)-\operatorname{dim} \mathbf{S}_{n} V$. The result follows using the fact that $\operatorname{dim} \mathbf{S}_{n} V=\left(\begin{array}{c}d+n-1 \\ n\end{array}\right)$.

We now turn to the proof of Theorems $\mathrm{C}$ and $\mathrm{D}$. Let $F$ be a free group of finite rank $e$, where $e \geq 2$. Let $R$ be a normal subgroup of $F$ such that $G=F / R$ is finite and $|G| \neq 1$. Let $V$ be the $\mathbb{Q} G$-module $\mathbb{Q} \otimes\left(R / R^{\prime}\right)$ and write $m=1+(e-1)|G|$. Note that, by Schreier's formula, $m$ is the rank of $R$. By [3, Theorem 1] (or by [11]), $R / R^{\prime}$ is faithful as $G$-module. Thus we can identify $G$ with a subgroup of GL $(V)$. As before we write $\chi(g)$ instead of $\chi_{V}(g)$.

PROOF OF THEOREM C. (i) It follows from the work of Gaschütz [11] that

$$
\chi(g)= \begin{cases}m & \text { if } g=1, \\ 1 & \text { if } g \in G \backslash\{1\} .\end{cases}
$$

In other words, $V$ is the direct sum of a one-dimensional trivial module and a free $\mathbb{Q} G$-module of rank $e-1$. Let $g \in G$ and let $q=|g|$. By (6),

$$
\begin{aligned}
H_{g}^{\prime} / H_{g} & =\sum_{n \geq 1} \chi\left(g^{n}\right) y^{n-1}=\sum_{\substack{n \geq 1 \\
q \mid n}} m y^{n-1}+\sum_{\substack{n \geq 1 \\
q \nmid n}} y^{n-1} \\
& =\sum_{k \geq 1}(m-1) y^{k q-1}+\sum_{k \geq 1} y^{k-1} \\
& =(m-1) y^{q-1}\left(1-y^{q}\right)^{-1}+(1-y)^{-1} .
\end{aligned}
$$

Since $H_{g}$ has 1 as its constant term, integration gives

$$
H_{g}=\left(1-y^{q}\right)^{-(m-1) / q}(1-y)^{-1} .
$$

Thus

$$
\begin{aligned}
H_{g} & =\left\{\sum_{i \geq 0}\left(\begin{array}{c}
-(m-1) / q \\
i
\end{array}\right)\left(-y^{q}\right)^{i}\right\}\left\{\sum_{j \geq 0} y^{j}\right\} \\
& =\left\{\sum_{i \geq 0}\left(\begin{array}{c}
((m-1) / q)+i-1 \\
i
\end{array}\right) y^{q i}\right\}\left\{\sum_{j \geq 0} y^{j}\right\} .
\end{aligned}
$$


Hence

$$
\chi_{\mathbf{s}_{n} v}(g)=\sum_{i=0}^{[n / q]}\left(\begin{array}{c}
((m-1) / q)+i-1 \\
i
\end{array}\right)
$$

But

$$
\left(\begin{array}{l}
j \\
0
\end{array}\right)+\left(\begin{array}{c}
j+1 \\
1
\end{array}\right)+\cdots+\left(\begin{array}{c}
j+s \\
s
\end{array}\right)=\left(\begin{array}{c}
j+s+1 \\
s
\end{array}\right)
$$

for all $j, s \geq 0$, as is easily proved by induction on $s$. Thus

$$
\chi_{\mathbf{s}_{n} V}(g)=\left(\begin{array}{c}
((m-1) / q)+[n / q] \\
{[n / q]}
\end{array}\right) .
$$

(ii) Suppose $n \geq 2$. If $g=1$ then $\chi_{\mathbf{M}_{n} V}(g)=(n-1)\left(\begin{array}{c}m+n-2 \\ n\end{array}\right)$ by Proposition 4.2. Thus we may assume that $q=|g| \neq 1$. By Proposition 4.1 (ii) with (8) and (9) we have

$$
M_{g}=-\left(1-y^{q}\right)^{-(m-1) / q}+y+1=-\sum_{j \geq 0}\left(\begin{array}{c}
((m-1) / q)+j-1 \\
j
\end{array}\right) y^{j q}+y+1
$$

This gives the remaining statements of (ii).

(iii) Suppose $n \geq 2$. We may work over the complex field $\mathbb{C}$ because, by [15, VII.1.21], the multiplicities in $\mathbf{M}_{n} V$ of the one-dimensional trivial $\mathbb{Q} G$ module and the regular $\mathbb{Q} G$-module are respectively equal to the multiplicities in $\mathbb{C} \otimes \mathbf{M}_{n} V$ of the one-dimensional trivial $\mathbb{C} G$-module and the regular $\mathbb{C} G$ module. Let $\phi$ be a complex irreducible character of $G$. The multiplicity of the corresponding irreducible module in $\mathbb{C} \otimes \mathbf{M}_{n} V$ is

$$
d_{n}(\phi)=\frac{1}{|G|} \sum_{g \in G} \phi(g) \overline{\chi_{\mathbf{M}_{n} V}(g)}
$$

where the bar denotes complex conjugation. Thus, by (ii),

$$
\begin{aligned}
|G| d_{n}(\phi)=\phi(1)(n-1) & \left(\begin{array}{c}
m+n-2 \\
n
\end{array}\right) \\
& -\sum_{\substack{q \mid(n,|G|) \\
q \neq 1}}\left(\sum_{\substack{g \in G \\
|g|=q}} \phi(g)\right)\left(\begin{array}{c}
(m+n-q-1) / q \\
n / q
\end{array}\right) .
\end{aligned}
$$

In particular, if $d_{n}$ denotes the multiplicity of the one-dimensional trivial module,

$$
|G| d_{n}=(n-1)\left(\begin{array}{c}
m+n-2 \\
n
\end{array}\right)-\sum_{\substack{q \mid(n,|G|) \\
q \neq 1}} v(q)\left(\begin{array}{c}
(m+n-q-1) / q \\
n / q
\end{array}\right)
$$


where $\nu(q)$ denotes the number of elements of $G$ of order $q$. For an arbitrary $\phi$, we have $|\phi(g)| \leq \phi(1)$ for all $g \in G$ (see [10, (6.7)]) and so, from (10),

$$
\begin{aligned}
|G| d_{n}(\phi) \geq \phi(1)(n-1)\left(\begin{array}{c}
m+n-2 \\
n
\end{array}\right) & \\
- & \sum_{\substack{q \mid(n,|G|) \\
q \neq 1}} v(q) \phi(1)\left(\begin{array}{c}
(m+n-q-1) / q \\
n / q
\end{array}\right) .
\end{aligned}
$$

Thus, from (11) and (12), $d_{n}(\phi) \geq \phi(1) d_{n}$ for all $\phi$. But the multiplicity of the irreducible module corresponding to $\phi$ in the free $\mathbb{C} G$-module of rank $d_{n}$ is exactly $\phi(1) d_{n}$. Thus $\mathbb{C} \otimes \mathbf{M}_{n} V$ has a free $\mathbb{C} G$-submodule of rank $d_{n}$. It has no larger free submodule because it contains the one-dimensional trivial module with multiplicity $d_{n}$.

(iv) Suppose that $n \geq 2$ and $(n,|G|)=1$. Again we may work over $\mathbb{C}$. By (10) and (11), $d_{n}(\phi)=\phi(1) d_{n}$ for every irreducible character $\phi$. Thus $\mathbb{C} \otimes \mathbf{M}_{n} V$ is a free $\mathbb{C} G$-module of rank $d_{n}$. Also, by (11),

$$
d_{n}=\frac{1}{|G|}(n-1)\left(\begin{array}{c}
m+n-2 \\
n
\end{array}\right)
$$

in this case.

PROOF OF THEOREM D. Let $d_{1}=e$ and for $n \geq 2$ let $d_{n}$ be defined as in Theorem C (iii). Since $F$ is finitely generated and $F / R$ is finite, $R$ is finitely generated and the groups $\gamma_{n} R / \gamma_{n+1} R$ and $\mu_{n} R / \mu_{n+1} R$ are finitely generated abelian groups. Note also that $\mu_{2} R=R^{\prime}$.

(i) We first prove that $Z\left(F / \mu_{n+1} R\right)=\left(\mu_{n} R / \mu_{n+1} R\right)^{G}$. Clearly we have $\left(\mu_{n} R / \mu_{n+1} R\right)^{G} \subseteq Z\left(F / \mu_{n+1} R\right)$. For the reverse inclusion let $f \mu_{n+1} R \in$ $Z\left(F / \mu_{n+1} R\right)$. Then, clearly, $f R^{\prime} \in Z\left(F / R^{\prime}\right)$. Since $R / R^{\prime}$ is a faithful $G$ module it follows that $f \in R$, and so $f \mu_{n+1} R \in Z\left(R / \mu_{n+1} R\right)$. Since $R$ is a free group of rank greater than $1, R / \mu_{n+1} R$ is a free group of rank greater than 1 in the variety of all groups which are both nilpotent of class $n$ and metabelian. The centre of such a group is equal to the $n$th term of its lower central series: see, for example, the remark after 35.22 of [18]. Thus

$$
Z\left(R / \mu_{n+1} R\right)=\gamma_{n}\left(R / \mu_{n+1} R\right)=\mu_{n} R / \mu_{n+1} R .
$$

It follows that $f \mu_{n+1} R \in \mu_{n} R / \mu_{n+1} R$ and so $f \mu_{n+1} R \in\left(\mu_{n} R / \mu_{n+1} R\right)^{G}$. Therefore $Z\left(F / \mu_{n+1} R\right)=\left(\mu_{n} R / \mu_{n+1} R\right)^{G}$. 
Let $E=R / R^{\prime \prime}$. Then $\mu_{n} R / \mu_{n+1} R \cong \gamma_{n} E / \gamma_{n+1} E$, and $\gamma_{n} E / \gamma_{n+1} E$ has the structure of a $\mathbb{Z} G$-module defined via conjugation. As noted in the proof of Proposition 2.10, $E / \gamma_{n+1} E$ is torsion-free. Thus $\gamma_{n} E / \gamma_{n+1} E$ and $\left(\gamma_{n} E / \gamma_{n+1} E\right)^{G}$ are free abelian groups (of finite rank). It remains to calculate the rank of $\left(\gamma_{n} E / \gamma_{n+1} E\right)^{G}$.

Let $U=E / \gamma_{2} E \cong R / \gamma_{2} R$. Since $U$ is a faithful $G$-module we can regard $G$ as a subgroup of $\mathrm{GL}(U)$. By Proposition $2.4, \gamma E$ has the structure of a $\mathbb{Z} \mathrm{GL}(U)$ module defined via endomorphisms of $E$, giving a $\mathbb{Z} G$-module structure to each $\gamma_{n} E / \gamma_{n+1} E$. It is easily verified that this is identical to the $\mathbb{Z} G$-module structure of $\gamma_{n} E / \gamma_{n+1} E$ defined via conjugation (because inner automorphisms of $F$ yield endomorphisms, indeed automorphisms, of $E$ ). By Proposition 2.8, $\gamma_{n} E / \gamma_{n+1} E \cong \mathbf{M}_{n} U$. Thus it suffices to find the rank of $\left(\mathbf{M}_{n} U\right)^{G}$. By a standard argument (see [13, Section 2]), this rank is equal to $\operatorname{dim}\left(\mathbb{Q} \otimes_{\mathbb{Z}} \mathbf{M}_{n} U\right)^{G}$. Let $V=\mathbb{Q} \otimes_{\mathbb{Z}} U$ : then $\mathbb{Q} \otimes_{\mathbb{Z}} \mathbf{M}_{n} U \cong \mathbf{M}_{n} V$, so it suffices to find $\operatorname{dim}\left(\mathbf{M}_{n} V\right)^{G}$. This is equal to the multiplicity of the one-dimensional trivial module in $\mathbf{M}_{n} V$. Hence, if $n \geq 2, \operatorname{dim}\left(\mathbf{M}_{n} V\right)^{G}=d_{n}$ by Theorem C (iii), and, if $n=1, \operatorname{dim}\left(\mathbf{M}_{n} V\right)^{G}=e$ by $(8)$.

(ii) Let $W=\mu_{n} R / \mu_{n+1} R$. Then

$$
\mu_{n} R /\left(\mu_{n} R, F\right) \mu_{n+1} R \cong W /[W, G]
$$

where $[W, G]$ denotes the subgroup of $W$ generated by $\{(g-1) w: g \in G, w \in$ $W\}$. By a standard argument (see [13, Lemma 2.1$]$ ), $W /[W, G]$ has the same torsion-free rank as $W^{G}$. Thus the result follows from (i).

\section{References}

[1] J. L. Alperin, Local representation theory (Cambridge Univ. Press, Cambridge, 1986).

[2] R. K. Amayo and I. Stewart, Infinite-dimensional Lie algebras (Noordhoff International, Leyden, 1974).

[3] M. Auslander and R. C. Lyndon, 'Commutator subgroups of free groups', Amer. J. Math. 77 (1955), 929-931.

[4] N. Bourbaki, Groupes et algèbres de Lie, Ch. I (Hermann, Paris, 1960).

[5] - Groupes et algebres de Lie, Ch. II (Hermann, Paris, 1972).

[6] A. Brandt, 'The free Lie ring and Lie representations of the full linear group', Trans. Amer. Math. Soc. 56 (1944), 528-536.

[7] R. M. Bryant and L. G. Kovács, 'Tensor products of representations of finite groups', Bull. London Math. Soc. 4 (1972), 133-135. 
[8] - 'Lie representations and groups of prime power order', J. London Math. Soc. (2) 17 (1978), 415-421.

[9] K.-T. Chen, 'Integration in free groups', Ann. of Math. (2) 54 (1951), 147-162.

[10] W. Feit, Characters of finite groups (Benjamin, New York, 1967).

[11] W. Gaschütz, 'Über modulare Darstellungen endlicher Gruppen, die von freien Gruppen induziert werden', Math. Z. 60 (1954), 274-286.

[12] J. A. Green, Polynomial representations of $G L_{n}$, Lecture Notes in Math. 830 (Springer, Berlin, 1980).

[13] N. D. Gupta, T. J. Laffey and M. W. Thomson, 'On the higher relation modules of a finite group', J. Algebra 59 (1979), 172-187.

[14] T. Hannebauer and R. Stöhr, 'Homology of groups with coefficients in free metabelian Lie powers and exterior powers of relation modules and applications to group theory', in: Proc. Second Internat. Group Theory Conference (Bressanone, 1989), Rend. Circ. Mat., Palermo, (2) Suppl. No 23 (1990) pp. 77-113.

[15] B. Huppert and N. Blackburn, Finite groups II (Springer, Berlin, 1982).

[16] M. Lazard, 'Sur les groupes nilpotents et les anneaux de Lie', Ann. Sci. École Norm. Sup. (3) 71 (1954), 101-190.

[17] I. G. Macdonald, Symmetric functions and Hall polynomials (Oxford Univ. Press, Oxford, 1979).

[18] H. Neumann, Varieties of groups (Springer, Berlin, 1967).

[19] A. L. Shmel'kin, 'Free polynilpotent groups', Izv. Akad. Nauk SSSR Ser. Mat. 28 (1964), 91-122 (in Russian).

University of Manchester Institute of Science and Technology

P.O. Box 88

Manchester, M60 1QD

United Kingdom
University of Manchester Institute of Science and Technology

P.O. Box 88

Manchester, M60 1QD

United Kingdom

Karl-Weierstrass-Institut für Mathematik

Mohrenstrasse 39

Postfach 1304

O-1086 Berlin

Germany 